\title{
Useful or Not? How Schizophrenic Patients Process the Relevance of a Visual Stimulus
}

\author{
Maxime Bubrovszky, Pierre Thomas \\ Department of Psychiatry, University Lille-Nord de France, Lille, France \\ E-mail: maxime.bubrovszky@chru-lille.fr \\ Received May 23, 2011; revised June 17, 2011; accepted June 30, 2011
}

\begin{abstract}
Introduction: The impairment of relevant information selection results in clinical symptoms of schizophrenia. Previous studies assessing visual modality, have reported an impairment of automatic sensory information processing related to abnormal irrelevant stimuli processing. In healthy subjects, neurophysiologic studies have distinguished two early posterior components which the second could be considered as a deviant processing marker. We propose to explore early selective properties of attention in acute patients using event related potential (ERP) methods. We hypothesize an impairment of the detection of deviant stimuli processing, supported by sensory integration neural region. Materiel and Method: Ten patients suffering from an acute episode of schizophrenia and ten controls were assessed with a simple three stimuli oddball paradigm analysed by principal components analysis (PCA) method which allows to separate overlapping components and to evaluate their modifications. Result: PCA distinguished two posterior negative components between 100 and $230 \mathrm{msec}$. The early one was not different between controls and patients. The later one was significantly decreased in the patients group. Discussion: Two different physiological components involved in stimuli detection were clearly isolated. The earliest, reflecting elementary perceptive process seems to be preserved in patients with acute schizophrenia whereas the later component, reflecting integrative processing involved in detection of deviance was impaired. These results could be a clue to understand clinical distractibility.
\end{abstract}

Keywords: Selective Attention, Vision, Event-Related Potentials, Schizophrenia, Principal Component Analysis

\section{Introduction}

The impairment of attentional and pre-attentional processes is associated with the incorrect selection of information in patients suffering from schizophrenia [1]. Using the visual mismatch negativity (vMMN) paradigm, Urban et al. showed an impairment of automatic sensory information processing among patients with schizophrenia in visual modality, as it was described in auditory modality [2]. These results suggest that the capacity of the patients to quickly detect deviant stimulus, as a first line of selection of information processing could be impaired. Recently, Kimura et al., exploring posterior negativity elicited by visual processing before $300 \mathrm{msec}$ in healthy subjects highlighted two distinct components: a forward one associated with refractory effect of visual cortex after stimulation and a later one considered as vMMN, index of deviance detection [3].

We propose here to study early stimuli processing in patients with schizophrenia to identify the level (elementary or integrative) of the alteration of visual processing in the disease. Since attentional selective properties may change during the course of the disease, we carefully selected patients suffering from acute schizophrenia.

We hypothesize that deviant detection process, supported by region involved in sensory integration should by more altered than the activity related to the refractory effect of the visual cortex. Using principal components analysis (PCA) on high density recording, we aimed to extract overlapping components from posterior negativity and reconstruct their sources [4,5]. Moreover, this analysis method should allow us to get information from a simple task, performable by acute patients. 


\section{Materiel and Method}

We recruited 10 patients in the acute phase of schizophrenia (as diagnosed by both a skilled clinician and a structured interview [SCID]) and 10 controls, according to the guidelines set by recommendations of the local ethics committee. All subjects were over the age of 18 . Groups were matched by age, sex and laterality (median for age and sex/laterality repartitions were not significantly different). Patients were included not later than one week after the introduction or modification of antipsychotic medications.

Visual 3-stimulus oddball paradigm was used, in which 500 stimuli were presented at random in the central visual field over $200 \mathrm{~ms}$ ("X" deviant, probability of appearance $p=0.1$, "o" target, $p=0.1$ and "O" standard, $p$ $=0.8$ ). Inter stimuli interval was between 800 and 1200 ms (pseudo random length). Subjects were asked to press a button as fast as possible when a target appeared. Electrophysiological signals were acquired with a 128 -electrodes cap, 10 - 20 extended system (Electrocap). Recordings (at a sampling rate of $1024 \mathrm{~Hz}$, impedances below $10 \mathrm{k} \Omega$, bandwith $1.10 \mathrm{e}-2$ to $45 \mathrm{~Hz}$ ) were recorded using an ANT Amplifier (Advanced NeuroTechnology, Netherlands).

Epochs of $800 \mathrm{~ms}$ were pre-defined (baseline before trigger: $100 \mathrm{~ms}$ ). In order to reject artefacts, signal variations of more than $100 \mu \mathrm{V}$ were excluded. This process was completed by visual inspection for artefacts exclusion [6]. We performed for the recordings of the 20 subjects temporal PCA followed by spatial PCA (based on covariance matrix with Varimax rotation [6]). This method is known to clearly distinguish different physiological activities [5]. We selected components with two criteria: posterior topography and latency before $300 \mathrm{~ms}$ (i.e., before the latency of the $\mathrm{P} 3$ complex, with ERP linked to attentional processing). Then we were able to to reconstruct the cortical sources of components from spatial factorial scores with sLORETA algorithm [4]. Factorial scores from PCA were used as variables for statistical tests (for each components Friedman test on three conditions (target/standard/deviant), then Mann-Withney for groups comparisons).

\section{Results}

All patients (mean age: 33.7 years Standard Deviation (S.D.) 8.7) received atypical antipsychotics (mean chlorpromazine equivalent: $290 \mathrm{mg} /$ day, S.D.:75). The mean PANSS score was 83 (S.D.: 22.4). Reaction times were longer for patients than for controls (medians: $438 \mathrm{~ms}$ and $387 \mathrm{~ms}, p=0.05$ ).

Five temporal components reported $91 \%$ of signal va- riance (Figure central panel). According to our a priori criteria, two components were withheld before $300 \mathrm{~ms}$. One temporal component reports alone more than $50 \%$ of variance from $185-232 \mathrm{~ms}$ and a second one from 103 - $150 \mathrm{~ms}$. Spatial PCA isolated a median posterior component (a) from the first temporal component (185 $232 \mathrm{~ms}$ ) Figure 1(a) and two symmetrical posterior components (b) and (c) for the second (103 - $150 \mathrm{~ms})$ Figures 1(b) and (c). Component (a) revealed sources at parieto-occipito-temporal (maximum for Brodman area 7 and 19, Figure 1(a)); components (b) and (c) showed more occipital sources (maximum for Brodman area 19 and 31, Figures 1(b) and (c)).

Non-parametrical statistical tests were performed on factorial scores from PCA (Kolmogorov tests for normality do not allow us to retain Gaussian distribution of variables). For the first component (a), a Friedman test on three conditions (target/standard/deviant) showed a significant difference and so confirmed the specificity of electrical activity evocated by the deviant condition $\left(\chi^{2}=\right.$ $6.632, d l=2, p=0.037$ [exact]).

The 2 groups differ significantly for this condition (Mann-Whitney test: $Z=-2.238, p=0.017$ [exact, bilateral]), but not for the target and standard conditions $(Z=$ $-0.898, p=0.4$ [exact, bilateral] and $Z=-0.245, p=$ 0.842 [exact, bilateral], respectively). These results confirm observation of grand average of event-related potential as shown on the left panel of the figure. Moreover, amplitude of event-related activity is lower in patient group. Earlier components were not condition-specific (Friedman test for B: $\chi^{2}=2 ; d l=2, p=0.417$ [exact] and for $\mathrm{C} \chi^{2}=1.684 ; d l=2, p=0.455$ [exact]). The groups did not differ for components (b) and (c).

\section{Discussion}

Compared to controls, patients showed an amplitude reduction of the component elicited by deviant condition, for which sources took place in occipito-temporal cortex. Thus, impairment of automatic sensory information processing already observed [2] would be more specifically related to pre-attentional detection of a deviant visual stimulus. The anatomical sources of this component shared a part of vMMN sources, close to areas of perceptual integration (Brodman 19, associative visual cortex, and 7, visuo-perceptive integration cortex). We clearly separate in latency and localization earlier components as Kimura did with a different method [3]. This activity corresponds to a refractory effect on the sensory cortex for Kimura et $a l$. The absence of difference between conditions and the source of the component in occipital cortex strengthens this hypothesis.

Links between our data and clinical features could be 


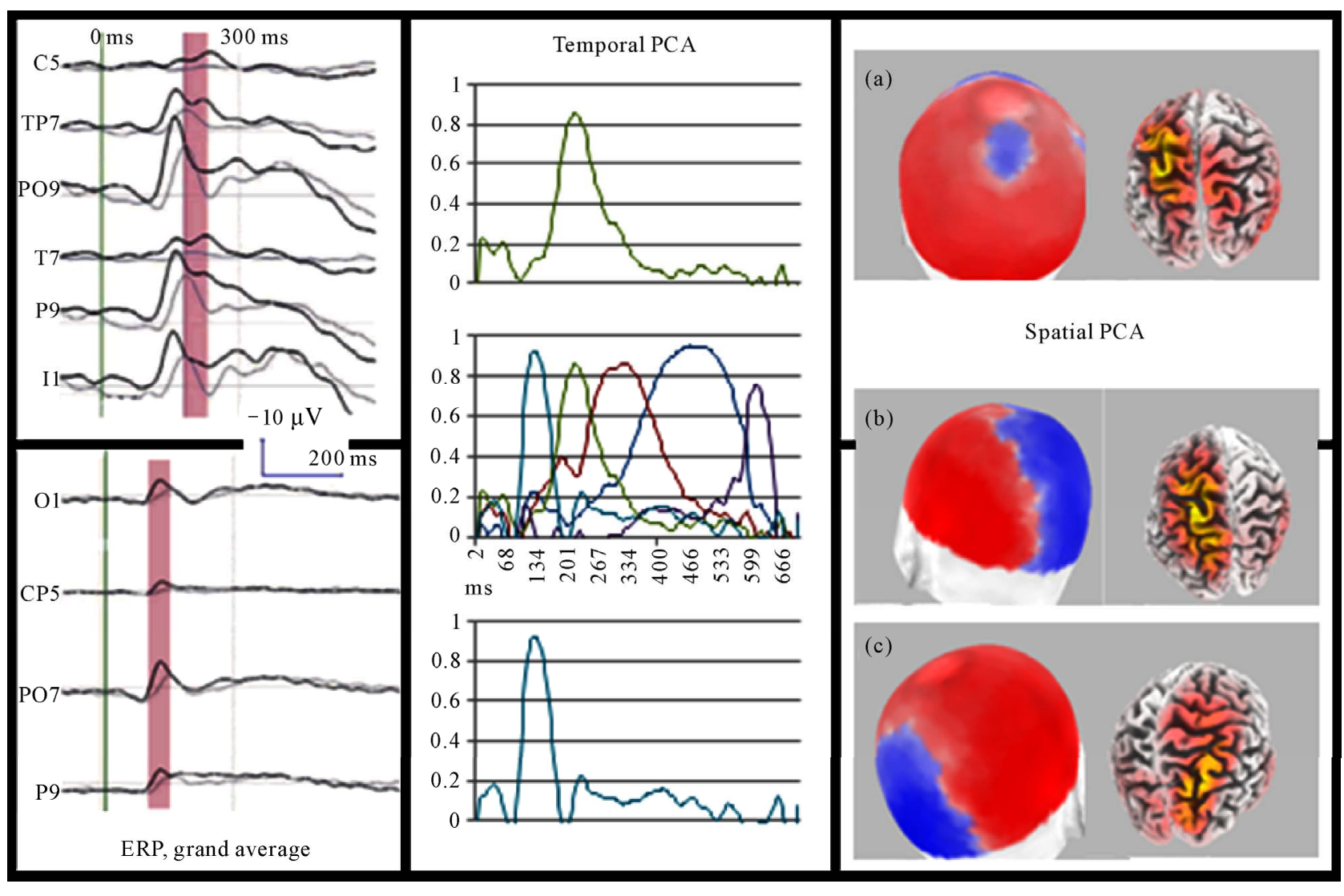

Figure 1. Left panel shows event related potential (grand average) for patients (grey lines) and controls (black lines) for posterior electrodes. Pink indicates temporal windows extracted by PCA (from central panel components). Center panel gives results from temporal PCA: eigenvalues (normalized) are represented function of time (ms). The components of interest are presented up (for the latest, reports alone more than $50 \%$ of variance from 185 - $232 \mathrm{~ms}$ ) and down (the earlier, reports alone more than $50 \%$ of variance from $103-150$ ms). Right panel shows scalp components extracted by spatial PCA from the temporal PCA components (the colors code for eigenvalues, normalized). For each one, cortical sources are given from sLORETA algorithm reconstruction.

Table 1. Statistical analysis for each component. Condition specificity appeared only for component A. For this component, groups differ significantly for deviant condition.

\begin{tabular}{ccccc}
\hline Components & $\begin{array}{c}\text { Friedman test for } \\
\text { conditions }\end{array}$ & $\begin{array}{c}\text { Groups comparison for } \\
\text { deviant condition }\end{array}$ & $\begin{array}{c}\text { Groups comparison for } \\
\text { target condition }\end{array}$ & $\begin{array}{c}\text { Groups comparison for } \\
\text { standart condition }\end{array}$ \\
\hline A $(185$ to $232 \mathrm{msec})$ & $\chi^{2}=6.632, p=0.037$ & $Z=-2.238, p=0.017$ & $Z=-0.898, p=$ N.S. & $Z=-0.245, p=$ N.S. \\
B $(103$ to $150 \mathrm{msec}$; left $)$ & $\chi^{2}=2, p=$ N.S. & $Z=-0.408, p=$ N.S. & $Z=-0.245, p=$ N.S. & $Z=-0.049, p=$ N.S. \\
C $(103$ to $150 \mathrm{msec}$; right $)$ & $\chi^{2}=1.684, p=$ N.S. & $Z=-1,715, p=$ N.S. & $Z=-0.082, p=$ N.S. & $Z=-0.08, p=$ N.S. \\
\hline
\end{tabular}

useful in understanding the distractibility and daily impairments of acute schizophrenia patients. Our study suggests that attentional dysfunction could be primarily related to early irrelevant processing impairment without perceptive alteration. Moreover these data brings elements for comprehension of early attentional orientation mechanism in visual modality. Finally, association of factorial analysis of electrophysiological data (here PCA) with sources reconstruction algorithm appears to be promising both for clinical and physiological exploration.

\section{References}

[1] D. L. Braff, "Information Processing and Attention Dysfunctions in Schizophrenia," Schizophrenia Bulletin, Vol. 19, No. 2, 1993, 233-59.

[2] A. Urban, J. Kremlacek, J. Masopust and J. Libiger, "Visual Mismatch Negativity among Patients With Schizophrenia," Schizophrenia Research, Vol. 102, No. 1, 2008, pp. 320-328. doi:10.1016/j.schres.2008.03.014

[3] M. Kimura, J. Katayama, H. Ohira and E. Schröger, "Vis- 
ual Mismatch Negativity: New Evidence from the Equiprobable Paradigm," Psychophysiology, Vol. 46, No. 2, 2009, pp. 402-409. doi:10.1111/j.1469-8986.2008.00767.x

[4] R. D. Pascual-Marqui, "Standardized Low-Resolution Brain Electromagnetic Tomography (sLORETA): Technical Details," Methods and Findings in Experimental and Clinical Pharmacology, Vol. 24, Suppl D, 2002, pp. 5-12.

[5] J. Dien, K. M. Spencer and E. Donchin, "Localization of the Event-Related Potential Novelty Response as Defined by Principal Components Analysis," Brain Research/Cognitive Brain Research, Vol. 17, No. 3, 2003, pp. 637-650. doi:10.1016/S0926-6410(03)00188-5

[6] T. W. Picton, S. Bentin, P. Berg, E. Donchin, S. A. Hillyard, R. Johnson, G. A. Miller, W. Ritter, D. S. Ruchkin, M. D. Rugg and M. J. Taylor, "Guidelines for Using Human Event-Related Potentials to Study Cognition: Recording Standards and Publication Criteria," Psychophysiology, Vol. 37, No. 2, 2000, pp. 127-152. doi:10.1111/1469-8986.3720127 